\title{
Aging Effects on Gut Microbiota in SAMP8 Mice ${ }^{\dagger}$
}

\author{
Lluïsa Miró 1,2,*, Miquel Moretó ${ }^{1}$, Concepció Amat ${ }^{1}$, Javier Polo ${ }^{2}$ and Anna Pérez-Bosque 1,* \\ 1 Departament de Bioquímica i Fisiologia, Facultat de Farmàcia i Ciències de l'Alimentació and Institut de \\ Nutrició i Seguretat Alimentària, Universitat de Barcelona (UB), 08028 Barcelona, Spain; \\ mmoreto@ub.edu (M.M.); camat@ub.edu (C.A.) \\ 2 APC-Europe S.L.U., 08403 Granollers, Spain; javier.polo@apc-europe.com \\ * Correspondence: lluisa.miro@ub.edu (L.M.); anna.perez@ub.edu (A.P.-B.) \\ + Presented at the The 1st International Electronic Conference on Nutrients - Nutritional and Microbiota \\ Effects on Chronic Disease, 2-15 November 2020; Available online: https://iecn2020.sciforum.net/.
}

Published: 30 October 2020

\begin{abstract}
We have studied the effects of aging on fecal microbiota composition in the senescenceaccelerated prone mice SAMP8 strain. We compared animals 2, 4 and 6 months old. Feces were collected at the end of each period and a genomic study was carried out on fecal DNA using the Illumina MiSeq analyzer. The Shannon diversity index showed similar values along this period and the number of species was neither affected by aging. The phylum Verrucobacteria went up with age, showing a 7 -fold increase at 6 months, compared to 2 months old mice. At family level, changes observed between 2 and 6 months of age involved significant increases in Bacteroidaceae $(q<0.001)$ and strong reductions in Lactobacillaceae $(q<0.0001)$ and Prevotellaceae $(q<0.05)$; at genus level, there was a significant reduction in probiotic Lactobacillus. At species level, we observed an agerelated reduction in Lactobacillus hayakitensis, a species involved in mucosal immune homeostasis, and in Blautia hansenii, which provides protection against Clostridium difficile infection. Interestingly, aging increases Parabacteroides goldsteiini, which is involved in the regulation of the TLR4 pathway. These results support the view that aging results in the proliferation of bacterial species that are associated with the immune deterioration of the gut mucosa.
\end{abstract}

Keywords: senescence; microbiota; aging; mice model of accelerated senescence

\section{Introduction}

The gut microbiota is a highly complex and diverse community of bacteria that closely interacts with the epithelium and the underlying immune cells in the gut [1]. The bacterial phyla that dominate the gut microbiota of different mammalian species are Bacteroidetes, Firmicutes, Actinobacteria and Proteobacteria [2,3]. In recent years, it has become clear that the gut microbiota has a major impact on the immune system, metabolism, and even behavior of the host [4]. Moreover, an imbalance in gut microbiota composition (dysbiosis) has been associated with several immunological, metabolic, and mental disorders [5].

Changes in the composition of the gut microbiota along aging likely contribute to immunosenescence and to the development of a pro-inflammatory phenotype [6]. In fact, elderly is more susceptible to infections and show a weaker immune protection after vaccination. Moreover, homeostasis between pro-inflammatory and regulatory responses is lost, which results in a state of low-grade chronic systemic inflammation, which is called inflammaging [7]. This age-related chronic inflammation likely contributes to the pathology of several diseases typically associated with aging such as dementia, stroke, and cardiovascular diseases. In addition, advanced age has been reported to increase intestinal permeability in rodents and non-human primates and may subsequently enhance translocation of luminal bacterial products and induce inflammation [8,9]. 
Inflammaging can impair brain function by an increase in inflammatory cytokine expression and elevated oxidative stress, blood-brain barrier breakdown, peripheral immune cell infiltration, and glial cell activation. Our results with the SAM model support this view because we have demonstrated that this mice model shows impaired intestinal immune response [10] as well as certain neurodegeneration that impairs memory function [11].

Aging-associated alterations in gut physiology (i.e., gastric motility disorders, hypochlorhydria, degenerative changes in enteric nervous system, etc.) have profound effects on the diversity, composition and functional features of intestinal microbiome [12]. These age-related differences can appear, in particular, because long-term stimulation of immune system may cause immunosenescence. With that, appears inflammaging, which accompanies many aging-associated diseases, including both gastrointestinal and non-gastrointestinal pathological conditions such as atherosclerosis, frailty, cancer, fatty liver disease, metabolic syndrome, type 2 diabetes, neurodegenerative diseases, etc. [12-14].

In previous studies performed with SAMP8 mice model, we have observed that at 6 months of age, mice exhibit an impaired immune response in the intestinal mucosa characterized by nonspecific gut-associated lymphoid tissue activation and a weak specific immune response [10]. In addition, in the same mice model, we have observed a cognitive impairment associated to increased brain inflammatory state and with a reduced blood-brain barrier [11].

Because this mouse model shows several characteristics of the deterioration associated with aging, both at the intestinal and cerebral levels, our aim in this work has been to determine whether these changes could be associated with an alteration in the profile of the microbiota.

\section{Materials and Methods}

\subsection{Animals}

Experiments were done in mice prone to senescence (SAMP8 strain), which were maintained and grown in the animal facility of the Facultat de Farmàcia i Ciències de l'Alimentació of the Universitat de Barcelona. Protocols used in this study were approved by the Animal Experimentation Ethics Committee (CEEA) of the Universitat de Barcelona, in accordance with the Generalitat de Catalunya's guidelines for the Care and Use of Laboratory Animals (DAAM: 7939 and 9272).

\subsection{Aging Effect on Microbiome Composition}

We used SAMP8 mice 2, 4 and 6 months old (groups named $2 \mathrm{M}, 4 \mathrm{M}$ and $6 \mathrm{M}$, respectively). Animals were maintained in conventional housing (3-4 animals per cage) until the age of 2 months, and from then they were kept in individual cages.

\subsection{Sample Obtention}

Feces were collected at three time points ( 2 months, 4 months and 6 months) in clean conditions and immediately frozen in liquid $\mathrm{N}_{2}$. Samples were maintained at $-80^{\circ} \mathrm{C}$ until use. DNA was extracted following Moretó et al. [15].

\subsubsection{S rDNA Gene Analysis}

The extracted genomic DNA was processed and the variable V3 and V4 regions of the 16S rRNA gene were amplified. The primers to detect $16 \mathrm{~S}$ rRNA gene were: $16 \mathrm{~S}$ forward $5^{\prime}$ TCGTCGGCAGCGTCAGATGTGTATAAGAGACAGCCTACGGGNGGCWGCAG-3' and reverse 5' -GTCTCGTGGGCTCGGAGATGTGTATAAGAGACAGGACTACHVGGGTATCTAATCC-3'. Highthrough sequencing was done using the Illumina MiSeq platform (Illumina, San Diego, CA, USA) at the Genomics and Bioinformatics Service, Universitat Autònoma de Barcelona (Bellaterra, Spain). 


\subsection{Statistics Analysis}

Results are presented as means \pm SEM. The statistical analysis was performed using GraphPad Prism $^{\circledast}$ software v 8.02 (GraphPad Software, Inc., La Jolla, CA, USA). Grubb's test was performed to determine outliers and the Shapiro-Wilk test was used to check the normality of data distribution. To compare $2 \mathrm{M}, 4 \mathrm{M}$ and $6 \mathrm{M}$ groups, the ANOVA test was used when data were normally distributed; otherwise the non-parametric Kruskal-Wallis test was carried out. All $p$-values were corrected for multiple testing using the false discovery rate (FDR) correction (Benjamini-Hochberg). Statistical differences were considered significant at $q<0.05$.

\section{Results}

\subsection{Shannon Diversity Index and the Number of Species}

The Shannon diversity index, which is commonly used to characterize species diversity in a community, was not changed by aging, nor was the number of species present in feces (Figure 1).

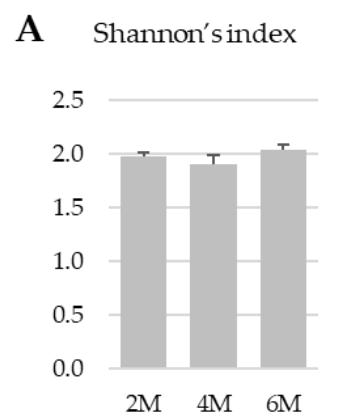

B Speciesidentified

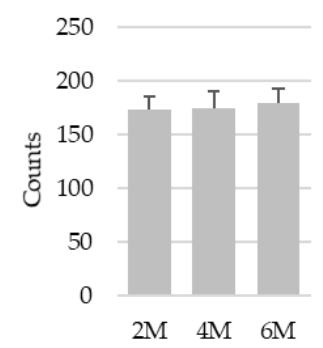

Figure 1. Microbial Shannon's index (A), number of species (B). Results are expressed as mean \pm SEM ( $n=11$ mice).

\subsection{Phylum Level}

The most represented phyla were Firmicutes (F) and Bacteroidetes (B) and this did not change with age (Figure 2). Therefore, the $\mathrm{F} / \mathrm{B}$ ratio was also similar in the three age groups, with mean values of $1.07(2 \mathrm{M}) ; 0.98(4 \mathrm{M})$ and $0.82(6 \mathrm{M})$. However, the phylum Verrucomicrobia increased severalfold with aging respect to $2 \mathrm{M}(4 \mathrm{M} q=0.004 ; 6 \mathrm{M} q=0.045)$.
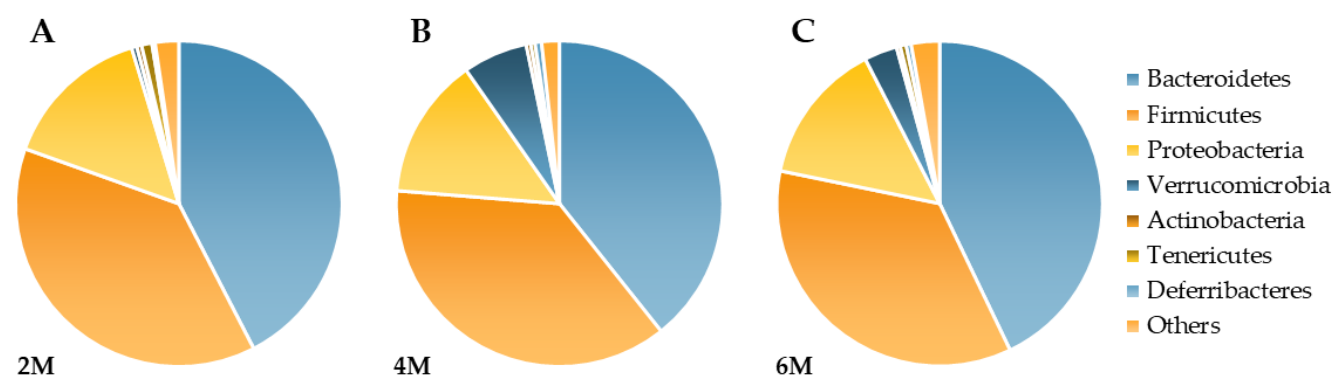

Figure 2. Effects of aging on fecal bacterial composition (Phylum level) at 2M (A), 4M (B) and 6M (C).

Results are expressed as percent of the total population at phylum level (mean) from 11 mice.

\subsection{Family Level Families}

At this taxonomic level there were changes in the relative presence of some between 2, 4 and 6 months of age (2M, $4 \mathrm{M}$ and $6 \mathrm{M}$, respectively; Figure 3). Four- and 6-months old mice showed increased relative abundance in Bacteroidaceae, Erysipelotrichaceae and Verrucomicrobiaceae (all $q$ $<0.02$ ). In contrast, both aged mice (4 and 6 months old) showed a pronounced reduction in the abundance of Prevotellaceae, Lactobacillaceae (both $q<0.001$ ). Other families were also modified by 
aging, smaller but significant reductions in Clostridiaceae and Helicobaceriaceae were also observed (all $q<0.05$ ).

A Bacteroidaceae

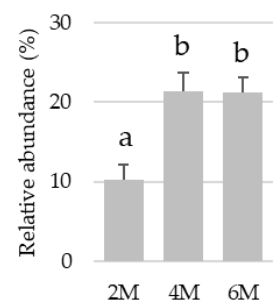

F Erysipelotrichaceae

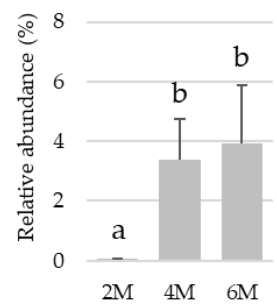

B Prevotellaceae

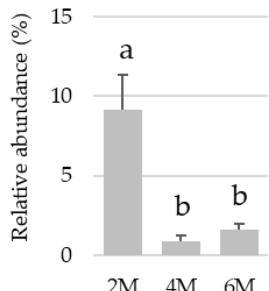

G Verrucomicrobiaceae

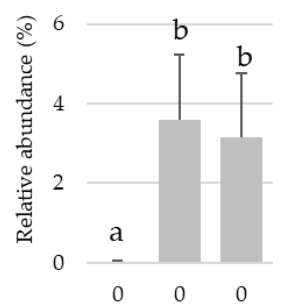

C Lactobacillaceae

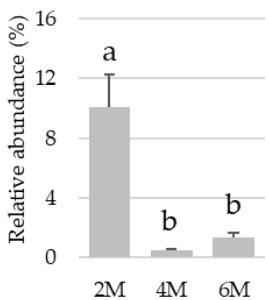

H Flavobacteriaceae

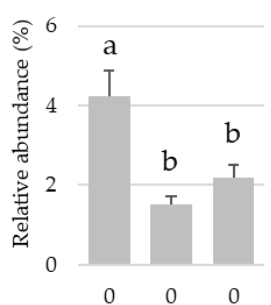

D Porphyromonadaceae

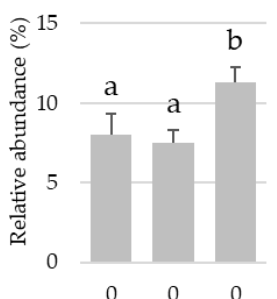

I Helicobacteraceae

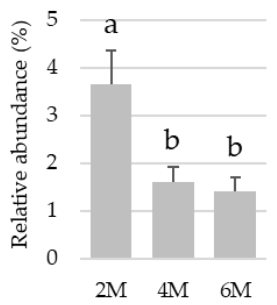

E Clostridiaceae

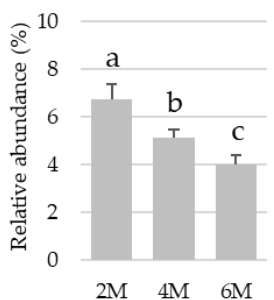

J Desulfovibrionaceae

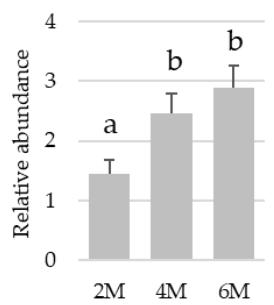

Figure 3. Effects of aging on fecal bacterial composition at family level. Results are expressed as percent of the total population at family level (mean \pm SEM; $n=10-11$ mice). Statistical differences were considered. significant at $q<0.05$ (corrected $p$ values).

\subsection{Genus Level}

There were important changes with age in the abundance of a number of genera, most of which showed a relative reduction in their abundance (Lactobacillus, Prevotella, Flavobacterium, Helicobacter, Natronincola, Odoribacter, Pedobacter, Olivibacter, just to mention those with a $1 \%$ presence of the Genus level; all $q<0.05$ ). Few genera (e.g., Bacteroides, Parabacteoides, Elysipelothrix, Butyricomonas, Akkermansia) showed an age-dependent increase (Table $1, q<0.05$ ).

Table 1. Effects of aging on fecal bacterial composition at genus level. Results are expressed as percent of the total population at genus level (mean \pm SEM; $n=10-11$ mice). Statistical differences were considered significant at $q<0.05$ (corrected $p$ values).

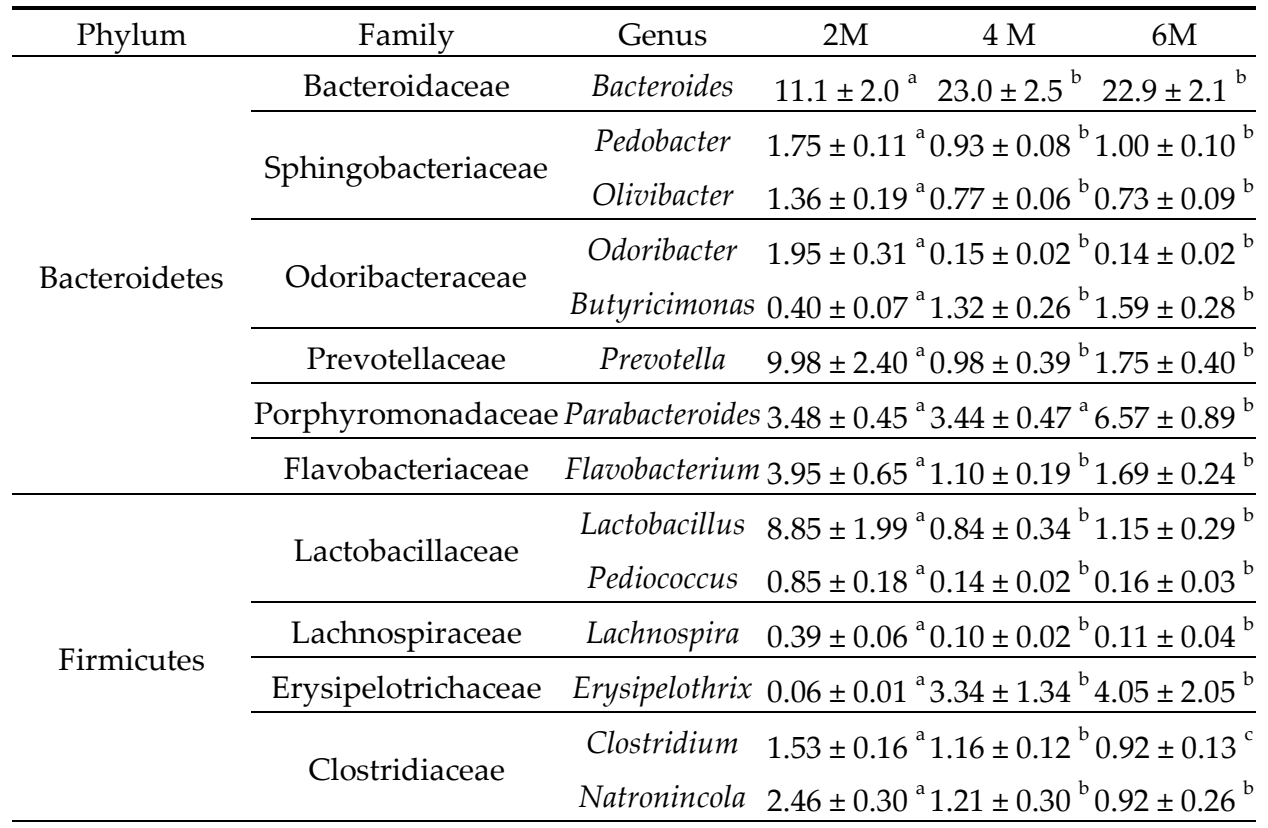




\begin{tabular}{cccc}
\hline \multirow{2}{*}{ Proteobacteria } & $\begin{array}{c}\text { Helicobacteraceae } \\
\text { Desulfovibrionaceae }\end{array}$ & Helicobacter & $3.92 \pm 0.76^{\mathrm{a}} 1.71 \pm 0.344^{\mathrm{b}} 1.53 \pm 0.32^{\mathrm{b}}$ \\
\hline Verrucomicrobia & Akkermansiaceae & Akkermansia & $1.53 \pm 0.26^{\mathrm{a}} 2.06 \pm 0.36^{\mathrm{b}} 3.10 \pm 0.43^{\mathrm{b}}$ \\
\hline
\end{tabular}

\subsection{Specie Level}

Several species from genus Bacteroides increased with aging $(q<0.05$; Table 2$)$, while other members of the phylum Bacteroidetes decreased, such as Odoribacter denticanis and Prevotella dentasini $(q<0.05)$. Respect to Lactobacillaceae family, several members of the Lactobacillus genus were reduced during aging, as well as Pediococcus argentinicus (all $q<0.05$ ). This behavior was also observed in two species included in the Lachnospiraceae family, Blautia hansenii and Johnsonella ignava (both, $q<0.05$ ). Instead, it has been observed a notable increase in Desulfovibrio psychrotolerans and Akkermansia municiphila (both, $q<0.05$ ), that belong to Proteobacteria and Verrucomicrobia phyla, respectively.

Table 2. Effects of aging on fecal bacterial composition at specie level. Results are expressed as percent of the total population at specie level (mean \pm SEM; $n=10-11$ mice). Statistical differences were considered significant at $q<0.05$ (corrected $p$ values).

\begin{tabular}{|c|c|c|c|c|}
\hline Phylum & Family & Species & $4 \mathrm{M}$ & $6 \mathrm{M}$ \\
\hline \multirow{10}{*}{ Bacteroidetes } & \multirow{6}{*}{ Bacteroidaceae } & Bacteroides xylanisolvens & $2.90 \pm 0.45^{\mathrm{a}} 7.94 \pm 0.90^{\mathrm{b}}$ & $6.62 \pm 1.16^{b}$ \\
\hline & & Bacteroides rodentium & $2.74 \pm 0.61^{\mathrm{a}} 7.49 \pm 1.52^{\mathrm{b}}$ & $3.16 \pm 0.90^{a}$ \\
\hline & & Bacteroides acidifaciens & $0.54 \pm 0.11^{\mathrm{a}} 5.94 \pm 0.96^{\mathrm{b}}$ & $4.11 \pm 0.70^{b}$ \\
\hline & & Bacteroides denticanum & $0.86 \pm 0.12^{\mathrm{a}} 3.98 \pm 1.36^{\mathrm{b}}$ & $6.55 \pm 1.11^{c}$ \\
\hline & & Bacteroides sartorii & $0.31 \pm 0.07^{\mathrm{a}} 1.26 \pm 0.25^{\mathrm{b}}$ & $1.08 \pm 0.29^{b}$ \\
\hline & & Bacteroides graminisolvens & $0.17 \pm 0.03^{\mathrm{a}} 0.89 \pm 0.12^{\mathrm{b}}$ & $1.02 \pm 0.18^{b}$ \\
\hline & \multirow{2}{*}{ Odoribacteraceae } & Odoribacter denticanis & $2.57 \pm 0.40^{\mathrm{a}} 0.22 \pm 0.06^{\mathrm{b}}$ & $0.20 \pm 0.03^{b}$ \\
\hline & & Butyricimonas virosa & $0.19 \pm 0.03^{\mathrm{a}} 1.30 \pm 0.22^{\mathrm{b}}$ & $0.85 \pm 0.20^{c}$ \\
\hline & Prevotellaceae & Prevotella dentasini & $16.1 \pm 3.73^{\mathrm{a}} 1.12 \pm 0.43^{\mathrm{b}}$ & $1.63 \pm 0.40^{b}$ \\
\hline & Porphyromonadaceae & Parabacteroides gordonii & $0.00 \pm 0.00^{\mathrm{a}} 0.01 \pm 0.01^{\mathrm{a}}$ & $3.57 \pm 0.81^{b}$ \\
\hline \multirow{10}{*}{ Firmicutes } & \multirow{5}{*}{ Lactobacillaceae } & Lactobacillus hayakitensis & $5.13 \pm 1.75^{\mathrm{a}} 0.33 \pm 0.13^{\mathrm{b}}$ & $0.86 \pm 0.21^{b}$ \\
\hline & & Lactobacillus taiwanensis & $1.93 \pm 0.67^{\mathrm{a}} 0.03 \pm 0.01^{\mathrm{b}}$ & $0.06 \pm 0.03^{b}$ \\
\hline & & Lactobacillus siliginis & $1.77 \pm 0.48^{\mathrm{a}} 0.19 \pm 0.07^{\mathrm{b}}$ & $0.28 \pm 0.06^{b}$ \\
\hline & & Lactobacillus intermedius & $0.85 \pm 0.44^{\mathrm{a}} 0.06 \pm 0.02^{\mathrm{b}}$ & $0.11 \pm 0.03^{b}$ \\
\hline & & Pediococcus argentinicus & $0.45 \pm 0.14^{\mathrm{a}} 0.02 \pm 0.01^{\mathrm{b}}$ & $0.02 \pm 0.01^{b}$ \\
\hline & \multirow{3}{*}{ Lachnospiraceae } & Blautia hansenii & $1.68 \pm 0.37^{\mathrm{a}} 0.65 \pm 0.07^{\mathrm{b}}$ & $0.95 \pm 0.23^{b}$ \\
\hline & & Ruminococcus gnavus & $0.24 \pm 0.06^{\mathrm{a}} 1.08 \pm 0.29^{\mathrm{b}}$ & $0.64 \pm 0.22^{\mathrm{ab}}$ \\
\hline & & Johnsonella ignava & $2.99 \pm 0.56^{\mathrm{a}} 0.31 \pm 0.06^{\mathrm{b}}$ & $0.41 \pm 0.11^{\mathrm{b}}$ \\
\hline & Erysipelotrichaceae & Erysipelothrix muris & $0.10 \pm 0.02^{a} 5.54 \pm 2.18^{b}$ & $1.66 \pm 0.83^{b}$ \\
\hline & Ruminococcaceae & Oscillospira guilliermondii & $1.03 \pm 0.12^{\mathrm{a}} 1.82 \pm 0.29^{\mathrm{b}}$ & $1.18 \pm 0.28^{a}$ \\
\hline \multirow{2}{*}{ Proteobacteria } & Helicobacteraceae & Helicobacter mastomyrinus & $4.13 \pm 0.64^{\mathrm{a}} 1.21 \pm 0.24^{\mathrm{b}}$ & $1.65 \pm 0.50^{b}$ \\
\hline & Desulfovibrionaceae & Desulfovibrio psychrotolerans & $s 0.26 \pm 0.06^{\mathrm{a}} 1.32 \pm 0.58^{\mathrm{ab}}$ & $2.31 \pm 0.48^{b}$ \\
\hline \multirow{2}{*}{ Verrucomicrobia } & Akkermansiaceae & Akkermansia muciniphila & $0.01 \pm 0.00^{\mathrm{a}} 7.17 \pm 2.48^{\mathrm{b}}$ & $4.75 \pm 2.20^{b}$ \\
\hline & Verrucomicrobiaceae & Luteolibacter algae & $0.01 \pm 0.00^{\mathrm{a}} 1.86 \pm 0.68^{\mathrm{b}}$ & $0.69 \pm 0.38^{a, b}$ \\
\hline
\end{tabular}

\section{Discussion}

Senescence is characterized by an increase in the basal inflammatory state, which contributes to the development and progression of various diseases associated with aging. This increase in the inflammatory state leads to a decrease in the functionality of the immune system and a reduced capacity to respond to the presence of infections or external aggressions, which is known as 
immunosenescence [16]. In addition, this decrease in the immune system functionality is accompanied by low-intensity inflammation sustained over time, known as inflammaging, which is characterized by increased production of pro-inflammatory mediators and cytokines $[17,18]$.

Dysbiosis observed during aging not only includes changes in the abundance of certain species and/or genera, which alone may already alter intestinal physiology, but pathogens may also appear. In the latter sense, the intestinal microbiota forms a stable community that reduces the invasion of non-native bacteria, because there is constant competition between normal bowel residents (commensals, mutualists and pathogens) [19].

In the microbiota of 6-month-old SAMP8 mice, the genera Bacteroides and Parabacteroides doubled in abundance. These results show the same pattern as that observed in a study of young and elderly individuals, where both genera accounted for $8-27 \%$ of the microbiome in a younger cohort, but more than $50 \%$ in the over 65 [20]. In addition, several studies have shown that the abundance of the genus Lactobacilli, which has generally been considered beneficial for intestinal health, decreases in the elderly [21]. In fact, lactobacillus loss is associated with fragility in the elderly [22]. In the 6-month SAMP8 mice from this study, a considerable loss in the abundance of different members of this genus was also observed, in line with studies performed in humans.

In previous studies, we have shown that at 6 months of age, SAMP8 mice exhibit an impaired immune response in the intestinal mucosa characterized by nonspecific gut-associated lymphoid tissue activation and a weak specific immune response [10]. Really, the gastrointestinal tract of the elderly is particularly susceptible to infectious diseases, and this is associated with a lower responsiveness of the mucosal immune system [23]. In addition, several studies have shown that aging is associated with an alteration in the composition of the intestinal microbiota, which can aggravate intestinal inflammation and in turn increase intestinal permeability [24]. In fact, the microbiota of older adults is less diverse [22], and immunosenescence and physiological changes at the intestinal level due to age can destabilize the microbiota [25]. Recently, it has been observed that an 'aged' microbiota promotes immune dysfunction, including increased systemic inflammation and phagocytosis of macrophages, which can be partially restored by transferring the microbiota from young mice to aged mice [26].

SAMP8 mice at 6-month-old showed an impaired intestinal permeability that in turns promote intestinal inflammation. Moreover, SAMP8 mice showed increased abundance of Akkermansia and Proteobacteria that have been associated with intestinal inflammation and permeability [27]. In addition, increased abundance of Akkermansia and Desulfovibrio (that belongs to proteobacteria phylum) are associated intestinal inflammation induced by DSS [28]. Further studies indicate that Desulfovibrio can contribute to intestinal inflammation comes from observations that these bacteria are associated with the pathogenesis of inflammatory bowel disease [29,30]. In fact, it is not surprising that these two genera have been associated to intestinal inflammation since both altered mucus structure that was more penetrable by bacteria leading to increased intestinal immune infiltration [31,32].

Research in recent years has linked intestinal epithelial barrier dysfunction to various neurological disorders. In fact, increased age-related intestinal inflammation and permeability are thought to contribute to the pathogenesis of these neurological disorders [33]. In this sense, the intestinal-brain axis and the intestinal microbiota appear as key actors, as they form an integrated network in which the microbiota and the central nervous system communicate through endocrine, immune, and neuronal signaling pathways [34]. Several translational studies show that microbiota transfer from patients with neurological disorders, including autism, multiple sclerosis, and Parkinson's disease, can influence behavior, motor dysfunction, and immune responses in different animal models [35-37].

It has been noted in gut of Alzheimer disease patients there is a decrease in the population of Firmicutes and an increase in the level of Bacteroidetes [38]. We did not observe a significant reduction in Firmicutes phylum, but we have observed a considerable reduction in several genera and species belonging to this phylum. In a similar manner, we also observed a huge increase in several species of Bacteroides that belongs to the Bacteroidetes phyla. These changes can be related to the increase in phosphorylated NF- $\mathrm{kB}$, a transcription factor that promotes inflammatory 
responses, observed in senescent SAMP8 mice, inducing neuroinflammation and memory loss [11]. In fact, Zhao and Lukiw [39] showed that LPS secreted by bacteria Bacteroides fragilis can activate the pro-inflammatory transcription factor $\mathrm{NF} \kappa \mathrm{B}$ in human primary microglial cells. Moreover, Cox et al., [34] found that Bacteroides correlates with aging and amyloid beta plaque deposition in brain of $\mathrm{Tg}$ 2576 mice, a model of Alzheimer disease.

SAMP8 mice, at 6 months of age, have also shown a sharp reduction in the Prevotellaceae family, which could also be related to the cognitive decline observed in these animals [11]. Several authors have also observed a similar reduction in this family in aged mice, which has been associated with neuropathology and Alzheimer's disease [40]. In fact, a reduction of Prevotellaceae can alter mucin synthesis and therefore increase mucosal permeability, which would allow local and systemic exposure to bacterial endotoxin, which can lead to the accumulation of $\alpha$-synuclein in the colon [41]. Aggregation-prone proteins such as $\beta$-amyloid and $\alpha$-synuclein can propagate from the intestine to the brain and contribute to the pathogenesis of Parkinson's disease, Alzheimer's disease, and other neurodegenerative disorders [42].

On the other hand, SAMP8 mice showed a sharp reduction in the abundance of several species and genera belonging to Firmicutes phylum, such as Lactobacillus or Blautia, which are the main members of the Lactobacilliaceae and Lachnospiraceae families, respectively. These two genders have been found to be reduced in the elderly and this reduction has been linked to the development of Alzheimer's disease [43,44]. Different strains of Lactobacillus produce acetylcholine and aminobutyric acid ( $\gamma$-Aminobutyric acid, GABA) and improve learning and memory in aged rats suffering from D-galactose-induced Alzheimer's disease [45], and the genus Blautia is positively correlated with a low inflammatory state and high cognitive scores [46].

Thus, we can conclude that in SAMP8 mice at 6 months of age a reduction of probiotic species is observed that modify the synthesis and secretion of neurotrophic factors, while the proinflammatory species of the intestinal microbiota increase, which they are able to secrete bacterial amyloids and lipopolysaccharides, which are considered neurotoxic [47]. In these mice we have previously described a deterioration in the barrier function of the intestinal wall and the blood-brain barrier. Therefore, these endotoxins can penetrate from the intestinal cavity into the bloodstream and into brain tissue, where they would have a negative effect on the structure and functions of the CNS and promote neuroinflammation and neurodegeneration.

\section{Conclusions}

6-month-old SAMP8 mice already show fecal microbiota dysbiosis consistent with the reduced intestinal barrier integrity and impaired intestinal immune response previously observed. Both, the dysbiosis observed in this work and the intestinal permeability could be involved in the neuroinflammation and blood-brain-barrier reduction that would lead to the cognitive decline observed in these mice.

Author Contributions: Conceptualization and design of the experiments, L.M., C.A., J.P. A.P.-B. and M.M.; methodology, L.M., C.A. and A.P.-B.; contribution with essential reagents and materials, J.P.; data analysis L.M., A.P.-B. and M.M.; writing-original draft preparation, A.P.-B. and M.M. writing-review and editing, C.A., A.P.-B. and M.M. All authors have read and agreed to the published version of the manuscript.

Funding: This study was funded by APC-Europe S.L.U. (Granollers, Spain) by research contracts with the Bosch i Gimpera Foundation of the University of Barcelona. The research group was also supported by grant 2017SGR945 for Consolidated Research Groups, Generalitat de Catalunya, Spain. L. Miró was supported by a grant from the Torres Quevedo programme (reference PTQ-12-05394) of the Spanish Ministerio de Economía y Competitividad.

Conflicts of Interest: C.A., A.P.-B., and M.M. declare that they have no conflict of interest; L.M. and J.P. are employed by APC-Europe S.L.U. The founding sponsors had no role in the design of the study; in the collection, analyses, or interpretation of data; in the writing of the manuscript, and in the decision to publish the results. 


\section{References}

1. Hooper, L.V.; Littman, D.R.; Macpherson, A.J. Interactions between the microbiota and the immune system. Science 2012, 336, 1268-1273, doi:10.1126/science.1223490.

2. Leser, T.D.; Amenuvor, J.Z.; Jensen, T.K.; Lindecrona, R.H.; Boye, M.; Møller, K. Culture-independent analysis of gut bacteria: The pig gastrointestinal tract microbiota revisited. Appl. Environ. Microbiol. 2002, 68, 673-690, doi:10.1128/AEM.68.2.673-690.2002.

3. Ley, R.E.; Bäckhed, F.; Turnbaugh, P.; Lozupone, C.A.; Knight, R.D.; Gordon, J.I. Obesity alters gut microbial ecology. Proc. Natl. Acad. Sci. USA 2005, 102, 11070-11075, doi:10.1073/pnas.0504978102.

4. Rescigno, M. Intestinal microbiota and its effects on the immune system. Cell. Microbiol. 2014, 16, 1004-1013, doi:10.1111/cmi.12301.

5. Carding, S.; Verbeke, K.; Vipond, D.T.; Corfe, B.M.; Owen, L.J. Dysbiosis of the gut microbiota in disease. Microb. Ecol. Health Dis. 2015, 26, 26191, doi:10.3402/mehd.v26.26191.

6. Vaiserman, A.M.; Koliada, A.K.; Marotta, F. Gut microbiota: A player in aging and a target for anti-aging intervention. Ageing Res. Rev. 2017, 35, 36-45, doi:10.1016/j.arr.2017.01.001.

7. Franceschi, C.; Capri, M.; Monti, D.; Giunta, S.; Olivieri, F.; Sevini, F.; Panourgia, M.P.; Invidia, L.; Celani, L.; Scurti, M.; et al. Inflammaging and anti-inflammaging: A systemic perspective on aging and longevity emerged from studies in humans. Mech. Ageing Dev. 2007, 128, 92-105, doi:10.1016/j.mad.2006.11.016.

8. Mullin, J.M.; Valenzano, M.C.; Verrecchio, J.J.; Kothari, R. Age- and diet-related increase in transepithelial colon permeability of Fischer 344 rats. Dig. Dis. Sci. 2002, 47, 2262-2270, doi:10.1023/A:1020191412285.

9. Tran, L.; Greenwood-Van Meerveld, B. Age-associated remodeling of the intestinal epithelial barrier. J. Gerontol. A Biol. Sci. Med. Sci. 2013, 68, 1045-1056, doi:10.1093/gerona/glt106.

10. Miró, L.; Garcia-Just, A.; Amat, C.; Polo, J.; Moretó, M.; Pérez-Bosque, A. Dietary animal plasma proteins improve the intestinal immune response in senescent mice. Nutrients 2017, 9, 1346, doi:10.3390/nu9121346.

11. Garcia-Just, A.; Miró, L.; Pérez-Bosque, A.; Amat, C.; Polo, J.; Pallàs, M.; Griñán-Ferré, C.; Moretó, M. Dietary Spray-Dried Porcine Plasma Prevents Cognitive Decline in Senescent Mice and Reduces Neuroinflammation and Oxidative Stress. J. Nutr. 2020, 150, 303-311, doi:10.1093/jn/nxz239.

12. Konturek, P.C.; Haziri, D.; Brzozowski, T.; Hess, T.; Heyman, S.; Kwiecien, S.; Konturek, S.J.; Koziel, J. Emerging role of fecal microbiota therapy in the treatment of gastrointestinal and extra-gastrointestinal diseases. J. Physiol. Pharmacol. 2015, 66, 483-491.

13. Bischoff, S.C. Microbiota and aging. Curr. Opin. Clin. Nutr. Metab. Care 2016, 19, 26-30, doi:10.1097/MCO.0000000000000242.

14. Pérez Martínez, G.; Bäuerl, C.; Collado, M.C. Understanding gut microbiota in elderly's health will enable intervention through probiotics. Benef. Microbes 2014, 5, 235-246, doi:10.3920/BM2013.0079.

15. Moretó, M.; Miró, L.; Amat, C.; Polo, J.; Manichanh, C.; Pérez-Bosque, A. Dietary supplementation with spray-dried porcine plasma has prebiotic effects on gut microbiota in mice. Sci. Rep. 2020, 10, 2926, doi:10.1038/s41598-020-59756-z.

16. Larbi, A.; Pawelec, G.; Wong, S.C.; Goldeck, D.; Tai, J.J.; Fulop, T. Impact of age on T cell signaling: A general defect or specific alterations? Ageing Res. Rev. 2011, 10, 370-378, doi:10.1016/j.arr.2010.09.008.

17. Franceschi, C.; Salvioli, S.; Garagnani, P.; de Eguileor, M.; Monti, D.; Capri, M. Immunobiography and the Heterogeneity of Immune Responses in the Elderly: A Focus on Inflammaging and Trained Immunity. Front. Immunol. 2017, 8, 982, doi:10.3389/fimmu.2017.00982.

18. Larbi, A.; Franceschi, C.; Mazzatti, D.; Solana, R.; Wikby, A.; Pawelec, G. Aging of the immune system as a prognostic factor for human longevity. Physiology 2008, 23, 64-74, doi:10.1152/physiol.00040.

19. Pickard, J.M.; Zeng, M.Y.; Caruso, R.; Núñez, G. Gut microbiota: Role in pathogen colonization, immune responses, and inflammatory disease. Immunol. Rev. 2017, 9, 70-89, doi:10.1111/imr.12567.

20. Claesson, M.J.; Cusack, S.; O’Sullivan, O.; Greene-Diniz, R.; de Weerd, H.; Flannery, E.; Marchesi, J.R.; Falush, D.; Dinan, T.; Fitzgerald, G.; et al. Composition, variability, and temporal stability of the intestinal microbiota of the elderly. Proc. Natl. Acad. Sci USA 2011, 108 (Suppl. 1), 4586-4591, doi:10.1073/pnas.1000097107.

21. Hopkins, M.J.; Sharp, R.; Macfarlane, G.T. Age and disease related changes in intestinal bacterial populations assessed by cell culture, $16 \mathrm{~S}$ rRNA abundance, and community cellular fatty acid profiles. Gut 2001, 48, 198-205, doi:10.1136/gut.48.2.198. 
22. Claesson, M.J.; Jeffery, I.B.; Conde, S.; Power, S.E.; O'Connor, E.M.; Cusack, S.; Harris, H.M.; Coakley, M.; Lakshminarayanan, B.; O'Sullivan, O.; et al. Gut microbiota composition correlates with diet and health in the elderly. Nature 2012, 488, 178-184, doi:10.1038/nature11319.

23. McDonald, K.G.; Leach, M.R.; Huang, C.; Wang, C.; Newberry, R.D. Aging impacts isolated lymphoid follicle development and function. Immun. Ageing 2011, 8, 1, doi:10.1186/1742-4933-8-1.

24. Rehman, T. Role of the gut microbiota in age-related chronic inflammation. Endocr. Metab. Immune Disord. Drug Targets 2012, 12, 361-367, doi:10.2174/187153012803832620.

25. Odamaki, T.; Kato, K.; Sugahara, H.; Hashikura, N.; Takahashi, S.; Xiao, J.Z.; Abe, F.; Osawa, R. Age-related changes in gut microbiota composition from newborn to centenarian: A cross-sectional study. BMC Microbiol. 2016, 16, 90, doi:10.1186/s12866-016-0708-5.

26. Thevaranjan, N.; Puchta, A.; Schulz, C.; Naidoo, A.; Szamosi, J.C.; Verschoor, C.P.; Loukov, D.; Schenck, L.P.; Jury, J.; Foley, K.P.; et al. Age-Associated Microbial Dysbiosis Promotes Intestinal Permeability, Systemic Inflammation, and Macrophage Dysfunction. Cell Host Microbe 2017, 21, 455-466, doi:10.1016/j.chom.2017.03.002.

27. Fransen, F.; van Beek, A.A.; Borghuis, T.; Aidy, S.E.; Hugenholtz, F.; van der Gaast-de Jongh, C.; Savelkoul, H.F.J.; De Jonge, M.I.; Boekschoten, M.V.; Smidt, H.; et al. Aged Gut Microbiota Contributes to Systemical Inflammaging after Transfer to Germ-Free Mice. Front. Immunol. 2017, 8, 1385, doi:10.3389/fimmu.2017.01385.

28. Håkansson, Å.; Tormo-Badia, N.; Baridi, A.; Xu, J.; Molin, G.; Hagslätt, M.L.; Karlsson, C.; Jeppsson, B.; Cilio, C.M.; Ahrné, S. Immunological alteration and changes of gut microbiota after dextran sulfate sodium (DSS) administration in mice. Clin. Exp. Med. 2015, 15, 107-120, doi:10.1007/s10238-013-0270-5.

29. Rowan, F.; Docherty, N.G.; Murphy, M.; Murphy, B.; Calvin Coffey, J.; O'Connell, P.R. Desulfovibrio bacterial species are increased in ulcerative colitis. Dis. Colon Rectum 2010, 53, 1530-1536, doi:10.1007/DCR.0b013e3181f1e620.

30. Mukhopadhya, I.; Hansen, R.; El-Omar, E.M.; Hold, G.L. IBD-what role do Proteobacteria play? Nat. Rev. Gastroenterol. Hepatol. 2012, 9, 219-230, doi:10.1038/nrgastro.2012.14.

31. Jakobsson, H.E.; Rodriguez-Pineiro, A.M.; Schutte, A.; Ermund, A.; Boysen, P.; Bemark, M.; Sommer, F.; Bäckhed, F.; Hansson, G.C.; Johansson, M.E. The composition of the gut microbiota shapes the colon mucus barrier. EMBO Rep. 2014, 16, 164-177, doi:10.15252/embr.201439263.

32. Ottman, N.; Davids, M.; Suarez-Diez, M.; Boeren, S.; Schaap, P.J.; Martins Dos Santos, V.A.P.; Smidt, H.; Belzer, C.; de Vos, W.M. Genome-scale model and omics analysis of metabolic capacities of Akkermansia muciniphila reveal a preferential mucin-degrading lifestyle. Appl. Environ. Microbiol. 2017, 83, 1014-1017, doi:10.1128/AEM.01014-17.

33. Walrath, T.; Dyamenahalli, K.U.; Hulsebus, H.J.; McCullough, R.L.; Idrovo, J.P.; Boe, D.M.; McMahan, R.H.; Kovacs, E.J. Age-related changes in intestinal immunity and the microbiome. J. Leukoc. Biol. 2020, doi:10.1002/JLB.3RI0620-405RR.

34. Cox, L.M.; Schafer, M.J.; Sohn, J.; Vincentini, J.; Weiner, H.L.; Ginsberg, S.D.; Blaser, M.J. Calorie restriction slows age-related microbiota changes in an Alzheimer's disease model in female mice. Sci. Rep. 2019, 9, 17904, doi:10.1038/s41598-019-54187-x.

35. Berer, K.; Gerdes, L.A.; Cekanaviciute, E.; Jia, X.; Xiao, L.; Xia, Z.; Liu, C.; Klotz, L.; Stauffer, U.; Baranzini, S.E.; et al. Gut microbiota from multiple sclerosis patients enables spontaneous autoimmune encephalomyelitis in mice. Proc. Natl. Acad. Sci. USA 2017, 114, 10719-10724, doi:10.1073/pnas.1711233114.

36. Sampson, T.R.; Debelius, J.W.; Thron, T.; Janssen, S.; Shastri, G.G.; Ilhan, Z.E.; Challis, C.; Schretter, C.E.; Rocha, S.; Gradinaru, V.; et al. Gut Microbiota Regulate Motor Deficits and Neuroinflammation in a Model of Parkinson's Disease. Cell 2016 167, 1469-1480.e12, doi:10.1016/j.cell.2016.11.018.

37. Sharon, G.; Cruz, N.J.; Kang, D.W.; Gandal, M.J.; Wang, B.; Kim, Y.M.; Zink, E.M.; Casey, C.P.; Taylor, B.C.; Lane, C.J.; et al. Human Gut Microbiota from Autism Spectrum Disorder Promote Behavioral Symptoms in Mice. Cell 2019, 177, 1600-1618.e17, doi:10.1016/j.cell.2019.05.004.

38. Cattaneo, A.; Cattane, N.; Galluzzi, S.; Provasi, S.; Lopizzo, N.; Festari, C.; Ferrari, C.; Guerra, U.P.; Paghera, B.; Muscio, C.; et al. Association of brain amyloidosis with pro-inflammatory gut bacterial taxa and peripheral inflammation markers in cognitively impaired elderly. Neurobiol. Aging 2017, 49, 60-68, doi:10.1016/j.neurobiolaging.2016.08.019.

39. Zhao, Y.; Lukiw, W.J. Bacteroidetes Neurotoxins and Inflammatory Neurodegeneration. Mol. Neurobiol. 2018, 55, 9100-9107, doi:10.1007/s12035-018-1015-y. 
40. Tran, T.T.T.; Corsini, S.; Kellingray, L.; Hegarty, C.; Le Gall, G.; Narbad, A.; Müller, M.; Tejera, N.; O’Toole, P.W.; Minihane, A.M.; et al. APOE genotype influences the gut microbiome structure and function in humans and mice: Relevance for Alzheimer's disease pathophysiology. FASEB J. 2019, 33, 8221-8231, doi:10.1096/fj.201900071R.

41. Forsyth, C.B.; Shannon, K.M.; Kordower, J.H.; Voigt, R.M.; Shaikh, M.; Jaglin, J.A.; Estes, J.D.; Dodiya, H.B.; Keshavarzian, A. Increased intestinal permeability correlates with sigmoid mucosa alpha-synuclein staining and endotoxin exposure markers in early Parkinson'sdisease. PLoS ONE 2011, 6, e28032, doi:10.1371/journal.pone.0028032.

42. Crews, L.; Tsigelny, I.; Hashimoto, M.; Masliah, E. Role of synucleins in Alzheimer's disease. Neurotox. Res. 2009, 16, 306-317, doi:10.1007/s12640-009-9073-6.

43. Albenberg, L.G.; Wu, G.D. Diet and the intestinal microbiome: Associations, functions, and implications for health and disease. Gastroenterology 2014, 146, 1564-1572, doi:10.1053/j.gastro.2014.01.058.

44. Zhuang, Z.Q.; Shen, L.L.; Li, W.W.; Fu, X.; Zeng, F.; Gui, L.; Lü, Y.; Cai, M.; Zhu, C.; Tan, Y.L.; et al. Gut Microbiota is Altered in Patients with Alzheimer's Disease. J. Alzheimers Dis. 2018, 63, 1337-1346, doi:10.3233/JAD-180176.

45. Junges, V.M.; Closs, V.E.; Nogueira, G.M.; Gottlieb, M.G.V. Crosstalk between gut microbiota and the central nervous system: A focus for Alzheimer's disease. Curr. Alzheimer Res. 2018, 15, 1179-1190. 10.2174/1567205015666180904155908.

46. Bajaj, J.S.; Hylemon, P.B.; Ridlon, J.M.; Heuman, D.M.; Daita, K.; White, M.B.; Monteith, P.; Noble, N.A.; Sikaroodi, M.; Gillevet, P.M. Colonic mucosal microbiome differs from stool microbiome in cirrhosis and hepatic encephalopathy and is linked to cognition and inflammation. Am. J. Physiol. Gastrointest. Liver Physiol. 2012, 303, G675-G685, doi:10.1152/ajpgi.00152.2012.

47. Askarova, S.; Umbayev, B.; Masoud, A.R.; Kaiyrlykyzy, A.; Safarova, Y.; Tsoy, A.; Olzhayev, F.; Kushugulova, A. The Links Between the Gut Microbiome, Aging, Modern Lifestyle and Alzheimer's Disease. Front. Cell. Infect. Microbiol. 2020, 10, 104, doi:10.3389/fcimb.2020.00104.

Publisher's Note: MDPI stays neutral with regard to jurisdictional claims in published maps and institutional affiliations.

(C) 2020 by the authors. Submitted for possible open access publication under the terms and conditions of the Creative Commons Attribution (CC BY) license (http://creativecommons.org/licenses/by/4.0/). 